\title{
Notas sobre el uso de la etnografia y la teoria fundamentada en ciencia politica. Un análisis amplio de la participación politica y el Estado en asentamientos informales de la periferia de Buenos Aires, Argentina ${ }^{1}$
}

\section{Patricio Bruno Besana ${ }^{2}$}

Universidad Nacional de San Martín, Argentina

patriciobesana@hotmail.com

Recibido: 12 de agosto de 2017

Aceptado: 18 de junio de 2018

Disponible en linea: 28 de diciembre de 2018

El presente es un artículo de reflexión finalizado en el marco de una beca postdoctoral, otorgada por el Consejo Nacional de Ciencia y Técnica (CONICET), dependiente del Estado Nacional de la República Argentina.

2 Doctor en Ciencia Política, Universidad Nacional de San Martín; Licenciado en Sociología, Universidad de Buenos Aires, y becario postdoctoral CONICET. Docente en la Escuela de Política y Gobierno, Universidad Nacional de San Martín, y miembro del Grupo Ambiente y Política. 


\title{
Notas sobre el uso de la etnografia y la teoria fundamentada en ciencia politica. Un análisis amplio de la participación politica y el Estado en asentamientos informales de la periferia de Buenos Aires, Argentina
}

\section{Resumen}

A la luz de los resultados obtenidos en el marco de una tesis doctoral sobre la participación política de quienes residen en asentamientos informales de la periferia de Buenos Aires (Argentina), el presente artículo reflexiona sobre la pertinencia del uso de la etnografia en la ciencia politica. Desde una perspectiva innovadora que la combina con el enfoque de la teoría fundamentada, se sugiere que dicha combinación permite avanzar a la teoría en temas poco desarrollados al interior de la disciplina. Puntualmente, se hace una introducción al tema de la participación política y se describen las ventajas del enfoque propuesto. Por último, se sugiere la posibilidad de complementar la etnografía y la teoría fundamentada con otros métodos y herramientas de recolección de datos.

Palabras clave: etnografia; ciencia política; participación política; etnografia virtual

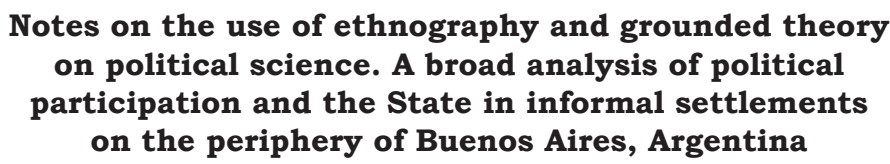

\begin{abstract}
By examining the results obtained in the framework of a doctoral thesis on the political participation of people in informal settlements in the periphery of Buenos Aires (Argentina), this article reflects on the relevance of the use of ethnography in political science. From an innovative perspective with an approach to grounded theory, it is suggested that this combination allows the theory to advance in less developed subjects within the discipline. More specifically, an introduction to the topic of political participation is made and the advantages of the proposed approach are described. Finally, the possibility of complementing ethnography and grounded theory with other tools and methods for data collection tools is suggested.
\end{abstract}

Keywords: ethnography; political science; political participation; virtual ethnography

\section{Anotações sobre o uso da etnografia e a teoria fundamentada em ciência politica. Uma análise ampla da participação política e do Estado em assentamentos informais da periferia de Buenos Aires, Argentina}

\begin{abstract}
Resumo
À luz dos resultados obtidos no âmbito de uma tese doutoral sobre a participação política de quem mora em assentamentos informais da periferia de Buenos Aires (Argentina), o presente artigo reflete sobre a pertinência do uso da etnografia na ciência politica. Desde uma perspectiva inovadora que a mistura com o enfoque da teoria fundamentada, sugere-se que tal combinação permite avançar à teoria em temas pouco desenvolvidos ao interior da disciplina. Pontualmente, uma introdução ao tema da participação política é feita e são descritas as vantagens do enfoque proposto. Por fim, sugere-se a possibilidade de complementar a etnografia e a teoria fundamentada com outros métodos e ferramentas de coleta de dados.
\end{abstract}

Palavras-chave: etnografia; ciência política; participação política; etnografia virtual 


\section{Introducción}

Mientras ciencias sociales como la antropología y la sociología incorporan a la etnografía como parte importante de sus corpus epistémicos, su aceptación en la ciencia política es aún materia de debate (Flick y Maeder, 2005; Shapiro et al., 2014). Durante los últimos años, algunas obras etnográficas fueron bien recibidas por la academia (Schatz, 2009; Weeden, 2012) e importantes publicaciones dedicaron varias páginas a discutir los beneficios que la etnografía podría traerle a la ciencia política (Box-Steffensmeier et al., 2008). Aun así, al mismo tiempo se publicaron obras influyentes que no la consideran siquiera como un método científico capaz de comprender ciertos aspectos de la realidad social (King et al., 2000; Gerber et al., 2014).

Hoy día, la etnografía continúa estando relegada a la hora de construir conocimiento en ciencia política, frente al uso de metodologías cuantitativas, fuentes históricas y bibliográficas, y herramientas como encuestas y entrevistas semiestructuradas. Dichos métodos, fuentes y herramientas a menudo son pertinentes para comparar y dar cuenta de las características que asumen instituciones y regímenes de gobierno, para analizar grandes procesos sociales del presente y del pasado, o para estudiar opiniones y discursos políticos. No obstante, los mismos presentan serias limitaciones para analizar comportamientos, acciones e incluso relaciones que mantienen cotidianamente distintos sujetos o actores sociales a nivel micropolítico.

En línea con perspectivas favorables a la investigación multimétodos, la triangulación de resultados y el pluralismo metodológico (Collier y Elman, 2008; Baubock et al., 2012; Della Porta y Keating, 2013), y con base en una investigación originalmente desarrollada en el marco de una tesis de doctorado (Besana, 2016), en el presente se sostiene que la etnografia es ideal para el avance de la teoría en ciencia política en temas que esta disciplina no ha podido elaborar satisfactoriamente. Puntualmente, se reflexiona sobre la pertinencia de la etnografia y se propone combinarla con la teoría 
fundamentada (Glaser y Strauss, 1967; Holton y Glaser, 2012) para analizar el tema de la participación politica a nivel micropolítico. Se argumenta que, en conjunto, la etnografia y la perspectiva de la teoría fundamentada permiten dar cuenta de formas de participación política que no pueden ser capturadas mediante otras perspectivas de análisis. De ese modo, la etnografia ayuda a ampliar y mejorar el entendimiento de un fenómeno complejo y multifacético como la participación política, permitiendo a la postre la elaboración inductiva de definiciones generales sobre las características que asumen las distintas formas en que participan ciertos actores o segmentos de la sociedad. A lo largo del artículo, se ejemplifica dicho argumento recurriendo al análisis realizado en una tesis doctoral sobre la participación política de quienes residen en asentamientos informales de la periferia de Buenos Aires, Argentina. Hacia el final del artículo, se muestra cómo una reconstrucción de las maneras en que participan dichos sectores permite avanzar sobre una caracterización de las formas en que se relacionan con el Estado y de las formas que asume este último en los ámbitos relevados. Al mismo tiempo, se reflexiona sobre la propia combinación entre etnografia y teoría fundamentada a fin de elaborar una teoría formal, y se sugiere que la etnografia puede ser complementada por etnografias en el campo virtual y por otras herramientas de investigación más estructuradas.

\section{Hacia una caracterización amplia de la participación política: aportes de la etnografia y la teoria fundamentada}

Como señalara hace tiempo el sociólogo italiano Alessandro Pizzorno (1975), la variedad de comportamientos o acciones que pueden ser considerados dentro de la categoría participación politica es sumamente amplia. Dichos comportamientos o acciones pueden incluir desde una charla de café donde una persona intenta convencer a otra de que su postura politica es la correcta, hasta la ejecución de 
un acto terrorista, pasando por las tareas que realizan a diario los políticos de carrera (Pasquino, 2011).

La dificultad conceptual en torno a la categoría participación política radica en qué se entiende por política y en dónde se sitúan sus límites. Si se circunscribe la política solo a los límites de la actividad de gobierno y a los actores que participan de ella, es más fácil brindar una definición de participación politica, pero se asume el costo de excluir una gran cantidad de acciones y a actores que, entre otras cosas, influyen directa o indirectamente, en la propia actividad de gobierno (Pizzorno, 1975). Si, por el contrario, se intenta incluir a la mayor cantidad de acciones y comportamientos posibles, la mayor dificultad conceptual radica en encontrar el o los elementos que los aúnan.

Desde principios del siglo XX, la ciencia política, en mayor medida, optó por acotar el análisis de la participación política a formas que se llevan a cabo a través de canales formales e institucionales (Pasquino, 2011). El predominio del institucionalismo formal en Estados Unidos hasta los años 30, y la revolución behaviorista que lo reemplazó luego (Murillo, 2017), sentaron "las bases científicas" de la disciplina (cf. Almond y Verba, 1963) y enfocaron su atención principalmente en acciones llevadas adelante por partidos politicos, comportamientos de políticos profesionales y el ejercicio del voto (Almond y Verba, 1963; Panebianco, 1990). En ese contexto, otras formas de participación política tales como protestas sociales y manifestaciones públicas, fueron analizadas principalmente por expertos de otras disciplinas, en su mayoría abocados al estudio de movimientos sociales, organizaciones de la sociedad civil y/o grupos de interés (Tilly, 2008; Collier y Handlin, 2009).

Aunque dicha división del objeto de estudio responde en parte a intereses específicos de cada disciplina e investigador; otra parte de ella responde a la pretensión de evitar las dificultades metodológicas que implican definiciones amplias de participación politica a la hora de capturar diversos comportamientos y acciones que no se 
expresan en el ámbito público y de los que no se tienen series sistemáticas de datos (Pasquino, 2011).

Ahora bien, ¿qué sucede cuando un analista está decidido a estudiar varias expresiones de la participación política? ¿Qué puede hacer al respecto? ¿Cómo puede definirla ampliamente? ¿Cómo puede capturar y analizar formas de participación que no tienen la suficiente publicidad, ni cuentan con datos recolectados sistemáticamente a los que pueda acceder? Finalmente, ¿cómo transformar la variedad de datos recabados en una teoría formal sobre la participación política? Aunque no parece haber respuestas que satisfagan completamente dichas preguntas, una opción es centrarse en algunos segmentos o actores de la sociedad y tratar de dar cuenta de la mayor cantidad de formas en que participan (vg. Collier y Handlin, 2009). Dicha opción se facilita cuando sus ámbitos de acción están circunscritos a un espacio determinado.

Para los análisis que se centran en el estudio de acciones localizadas, situadas en territorios sociales específicos, abordables a escala micro, la etnografía parece ser la opción más adecuada. En primer lugar, porque solo la etnografia permite capturar directamente las acciones de los sujetos que participan in situ, mediante la observación participante del investigador (Migdal, 2009). En segundo lugar, porque la etnografia es de gran utilidad cuando se pretende pensar ampliamente en términos que son al mismo tiempo categorias nativas de sujetos socialmente situados y parte del vocabulario del investigador que los aborda (Balbi y Boivin, 2008). En ese sentido, la etnografia permite dotar de contenido a términos abstractos, polisémicos y ambiguos como "política", "participación política" y "Estado", evitando que dichos contenidos resulten exclusivamente de la especulación teórico-normativa de quien investiga y reconstruyendo, para ello, las experiencias vividas por actores socialmente situados y los significados que atribuyen a dichas experiencias.

Considerada como un "diálogo" autoconsciente entre el etnógrafo y los sujetos que aborda, así como un proceso crítico de 
generación de conocimiento (Guber, 2001; Balbi y Boivin, 2008), la etnografia se presenta como fundamental para visibilizar formas de participación que no son consideradas como tales y contrarrestar la tendencia de la ciencia política a tratar a su objeto de estudio como un campo o una esfera especializada y claramente delimitada a las actividades de gobierno que desarrollan ciertos actores en un entramado de instituciones presentadas como si fueran "La" política (Balbi y Boivin, 2008).

Ahora bien, si la etnografia es una estrategia adecuada para definir ampliamente la participación política, así como para capturar y analizar diversas formas de participación que no tienen suficiente publicidad, ni cuentan con datos recogidos sistemáticamente, aquí se plantea otra dificultad: cómo transformar la información elaborada etnográficamente mediante un análisis de caso en una teoría formal con cierto grado de generalización. Aunque las posibilidades de generalizar la información elaborada etnográficamente son objeto de debate entre disciplinas (Quirós, 2011), lo cierto es que la etnografia puede valerse para ello de la teoría fundamentada, justamente concebida para construir teorías formales a partir de datos que se recaban directamente del análisis social (Glaser y Strauss, 1967).

En la práctica, la teoría fundamentada se basa en dos estrategias: el proceso de comparación continua o método comparativo constante y el muestreo teórico. El primero supone que los datos recabados se van categorizando, comparando y analizando simultánea y continuamente (Cuñat Giménez, 2007). Esta estrategia facilitaría la elaboración de categorías con distintos niveles de abstracción que emergen directamente de los datos. Básicamente, se comienza con categorías guías que refieren al fenómeno que se pretende estudiar, para pasar sucesivamente a categorías emergentes de bajo, medio y alto nivel de abstracción (Holton y Glaser, 2012). Las primeras categorías, como su nombre lo indica, son definiciones amplias que tienen por objeto guiar la investigación en su primera etapa (o etapa de descubrimiento), hasta ser elaboradas con fundamento en los datos. Estas categorías están abocadas a descubrir 
procesos sociales básicos a partir de los cuales construir categorías emergentes (Glaser y Strauss, 1967). Hasta tanto no se descubran dichos procesos, se recomienda no hacer una revisión bibliográfica a fin de evitar ceñir los resultados de la investigación a un marco conceptual acotado. En consecuencia, las categorías guías pueden surgir de ideas o intuiciones del investigador, así como de preconceptos u opiniones ampliamente diseminadas en la sociedad. Para construirlas pueden utilizarse definiciones negativas encargadas de establecer una categoría a partir de decir lo que no es; definiciones ambi o multivalentes que remiten a varios preconceptos, perspectivas o ideas a veces contrapuestas; o a una combinación de ambas.

La idea de las categorías guías es que acoten lo menos posible el tema de estudio con objeto de obtener indicadores diversos que puedan ser agrupados según sus singularidades y/o regularidades en categorías emergentes. Una vez elaboradas las primeras categorías emergentes, se continúa recabando y comparando la información con el objeto de: 1) enriquecer el contenido de las mismas; 2) establecer relaciones entre dichas categorías, y 3) generar nuevas categorías de mayor nivel de abstracción. Cuando esto deja de suceder, se da por finalizada la muestra. A este tipo de muestras se las denomina muestras teóricas y se diferencian de las estadísticas porque su finalización no depende del tamaño de la misma, sino del principio de saturación teórico (Cuñat Giménez, 2007). Por este, se entiende al momento en que nueva información recabada ya no produce nuevo contenido teórico (Holton y Glaser, 2012). Según sus propios mentores, la teoría fundamentada no es en realidad una teoría, sino un método que hace emerger a la teoría de los datos (Glaser y Strauss, 1967). Este artículo, en cambio, propone pensarla de modo más amplio, como una estrategia, actitud o predisposición para hacer emerger teoria directamente del proceso de análisis social. En ese sentido, una etnografia puede desarrollarse con la predisposición de la teoría fundamentada con toda facilidad, puesto que el etnógrafo también elige con criterios teóricos centrarse en ciertos eventos, personas y relaciones sociales (y no en otras) y construye conocimiento mediante su continua comparación (Quirós, 2011), así como sucede con el muestreo teórico y el proceso de comparación constante. 
Partiendo de ideas básicas e intuitivas, antes que de marcos conceptuales rígidos, la etnografía presenta algunas facilidades para hacer progresar a la teoría que resultan del diálogo y la menor distancia metodológica entre el investigador y los sujetos abordados. De forma similar, el largo periodo de tiempo que insume el desarrollo de una estrategia etnográfica permite al investigador generar lazos de confianza y trascender las barreras sociales que lo separan de los sujetos estudiados. Flanquear esas barreras sociales permite acceder a sitios, situaciones y testimonios usualmente vedados a extraños pero habituales entre dichos sujetos. El acceso a los "circuitos de intimidad" de los sujetos (Herzfeld, 2004) suele resultar en la elaboración de conceptos o categorías emergentes más complejas y con un mayor grado de abstracción que los que pueden generarse por medio de herramientas que solo capturan datos superficiales o visibles de un fenómeno.

A continuación, se describen algunas características de la población que reside en asentamientos informales de la periferia de Buenos Aires. Además, se presenta información de primera mano sobre el asentamiento analizado bajo la perspectiva etnográfica propuesta.

\section{Asentamientos informales, caracteristicas y población}

Llamados slums o shantytowns en la literatura de habla inglesa (Davis, 2007), espacios de segregación urbana en la literatura influenciada por la escuela francesa (Wacquant, 2007), la etiqueta asentamientos informales denomina aquí al fenómeno de ocupaciones ilegales de tierras en Argentina (Techo, 2016). En general, quienes residen en asentamientos informales carecen de un título de propiedad y de una o varias de las siguientes condiciones: viviendas de materiales duraderos, espacio suficiente para todos los miembros que conforman un hogar, acceso a fuentes mejoradas de agua e instalaciones de saneamiento (UN-Habitat, 2012). 
En Argentina, la población que reside en asentamientos informales no paró de aumentar desde principios de los 80 en adelante. En la provincia de Buenos Aires creció ininterrumpidamente entre 1981 y 2007, alcanzando un incremento del $220 \%$ sobre un incremento total de la población de tan solo el $35 \%$ (Cravino et al., 2007). Actualmente, se calcula que una de cada 10 personas en Argentina vive en asentamientos informales, aproximadamente el $61 \%$ de ellas lo hace en asentamientos de la provincia de Buenos Aires (Techo, 2016).

Factores de distinta índole someten a dicha población a condiciones de extrema pobreza y de alta degradación ambiental, dos problemáticas que se refuerzan mutuamente (Curutchet et al., 2012). Por un lado, el escaso poder adquisitivo de vastos sectores populares los obliga a localizarse en tierras con poco o nulo valor comercial, mayormente en áreas inundables y/o expuestas a riesgos ambientales tales como: contaminación por vuelcos industriales, descargas cloacales, basurales "a cielo abierto", cursos de agua degradados, entre otros. Por otro lado, la falta o inadecuada provisión de servicios de agua potable y cloacas, barrido, limpieza y recolección de residuos, energía eléctrica, gas, etc., se convierten en un agravante de las condiciones ambientales que ya de por sí presentan los asentamientos.

Al respecto, se estima que solo el $12 \%$ de la población que reside en asentamientos informales de Buenos Aires cuenta con conexiones formales de agua potable de red, un $5.2 \%$ es servida por redes cloacales y un $44.4 \%$ por redes de energía eléctrica (Techo, 2016). En este último caso, el mayor problema consiste en la mala calidad del servicio y los riesgos que eso implica (Techo, 2016).

Dichos datos son complementarios a los obtenidos por otros estudios que muestran cómo el acceso a servicios públicos aumenta si se incluyen en la estadística canales de acceso informales a los mismos (Curutchet et al., 2012), tales como algunas de las formas de participación que se verán más adelante. 
Una encuesta que data de 2011, realizada a 220 vecinos del asentamiento etnografiado, muestra cómo acceden a distintos servicios públicos, aunque en condiciones de informalidad y de gran precariedad. Según datos arrojados por la misma encuesta, el acceso a servicios de electricidad, recolección de basura y agua corriente es alto (99\%, $90.4 \%$ y $87.6 \%$ respectivamente), aunque precario. Según los encuestados, dichos servicios se encuentran entre los mayores problemas que deben sortear a diario (ver figura 1). A dichos problemas se suma la contaminación de un brazo del valle de inundación del Río Reconquista, el segundo más contaminado de la Argentina (Defensoría del Pueblo et al., 2007), al norte de la periferia de Buenos Aires sobre el que se localiza el asentamiento y al que los vecinos denominan "Zanjón".

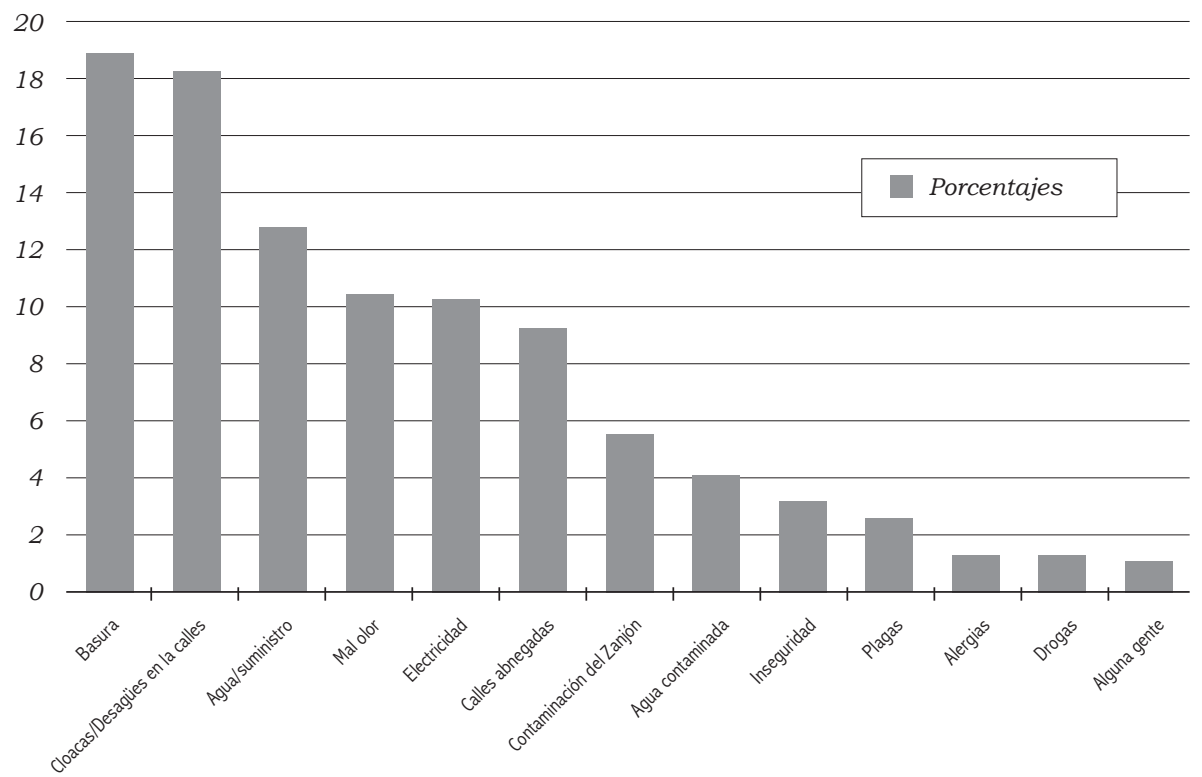

Figura 1. Problemas más importantes según encuestados Fuente: coelaboración del autor con datos de la Encuesta de Consumo Energético 2011, financiada por la Fundación Bariloche en el marco del proyecto PIP $11220090100079 .^{3}$

3 Agradezco a los directores de proyectos PIP-Gustavo Curutchet, Ricardo Gutiérrez y Silvia Grinberg-por permitirme utilizar datos elaborados con financiamiento de los mismos. 
En términos socioeconómicos, el nivel de ingresos de los 220 vecinos encuestados estaba por debajo de la línea de pobreza estipulada por el Instituto Nacional de Estadísticas y Censos (INDEC) en el momento en que se realizó la encuesta. Respecto a sus fuentes de ingresos, la mayoría de los encuestados contaba con un empleo informal o con ingresos provenientes del financiamiento público provisto por programas de transferencia condicionada (ver tabla 1). Dichos programas fomentan el cooperativismo y la conformación de organizaciones de vecinos, encargadas de desarrollar labores vinculadas a la prestación de bienes y servicios públicos, a cambio de una pequeña transferencia monetaria.

\begin{tabular}{|c|c|c|c|c|c|c|c|c|}
\hline $\begin{array}{c}\text { Empleado } \\
\text { /obrero }\end{array}$ & $\begin{array}{c}\text { Financiamiento } \\
\text { público }\end{array}$ & $\begin{array}{c}\text { Cuenta } \\
\text { propia }\end{array}$ & Changas & Cartonero & $\begin{array}{c}\text { Venta } \\
\text { ambulante }\end{array}$ & Otra & Desocupado & $\begin{array}{c}\text { No } \\
\text { responde }\end{array}$ \\
\hline $28,1 \%$ & $19,4 \%$ & $18,2 \%$ & $4,3 \%$ & $3,8 \%$ & $1,0 \%$ & $11,0 \%$ & $12,9 \%$ & $1,4 \%$ \\
\hline
\end{tabular}

Tabla 1. Fuentes de ingresos de los vecinos de Santa María Fuente: coelaboración del autor con datos de la Encuesta de Consumo Energético 2011 financiada por la Fundación Bariloche en el marco del proyecto PIP 11220090100079.

Algunos de los datos aquí presentados se relacionan con las formas de participación reveladas en el proceso de investigación. Antes de centrarse en ellas, se repasan dos grandes visiones que históricamente discutieron sobre las formas en que los pobres participan políticamente.

\section{La participación dividida: entre el clientelismo y los movimientos sociales}

Durante años, los trabajos abocados a analizar la participación política de los más pobres tendieron a reducirla a las formas en que se manifiestan en movimientos sociales o a las relaciones que desarrollan al interior de redes clientelares (eg. Piven y Cloward, 2012; Stokes et al., 
2013). Mientras la literatura sobre movimientos sociales caracterizó la protesta social frente al Estado como la forma de participación política predominante de los pobres para obtener bienes y servicios colectivizados o públicos (eg. Svampa y Pereryra, 2005), la literatura referida al clientelismo hizo lo propio con las relaciones entre brokers (en Argentina denominados punteros) y clientes, basadas en intercambios discrecionales de apoyo politico por favores individuales (Zarazaga, 2013). De ese modo, en los primeros análisis lo colectivo aparece como una marca de los sujetos que participan, los intereses que los impulsan a participar, las formas en que participan y los bienes y servicios que persiguen; mientras que, en los segundos, sucede lo contrario: lo individual aparece como una marca de esos mismos elementos (en la tabla 2 se contraponen ambos tipos de análisis).

\begin{tabular}{|c|c|c|}
\hline Elementos & \multicolumn{2}{|c|}{ Visiones sobre la participación política de los pobres } \\
\hline Literaturas & Movimientos sociales & Clientelismo \\
\hline Actores & $\begin{array}{c}\text { Integrantes de movimientos } \\
\text { (iguales entre si) }\end{array}$ & $\begin{array}{c}\text { Patrón politico, Brokers o } \\
\text { punteros, clientes }(\mathrm{c} / \text { jerarquías } \\
\text { distintas) }\end{array}$ \\
\hline $\begin{array}{c}\text { Formas de } \\
\text { participación }\end{array}$ & Protestas sociales & Intercambios individuales \\
\hline $\begin{array}{c}\text { Bienes o } \\
\text { servicios } \\
\text { perseguidos }\end{array}$ & Colectivizados o públicos \\
\hline $\begin{array}{c}\text { Identidades o } \\
\text { intereses que } \\
\text { lo impulsan }\end{array}$ & Identidades comunes & Intereses egoístas \\
\hline
\end{tabular}

Tabla 2. Elementos de la participación según el foco analítico en los movimientos sociales o en el clientelismo Fuente: elaboración propia.

Teóricamente, la oposición entre movimientos sociales y redes clientelares giró en torno a las identidades comunes $e$ intereses egoístas como variables independientes que impulsarian a los pobres a participar en los primeros o en las segundas, excluyentemente (Vommaro y Quirós, 2011). Sin embargo, dichos elementos aparentan estar más relacionados a los métodos de análisis 
utilizados y a supuestos onto-epistemológicos de ambas literaturas que a datos empíricos.

Mientras las identidades comunes que impulsarian a los integrantes de movimientos sociales a actuar colectivamente resultan sobredimensionadas por el acercamiento de la literatura al objeto de análisis, principalmente cuando este se hace visible mediante manifestaciones públicas (vg. Tilly, 2008), los intereses egoístas que impulsarian a los pobres a participar de relaciones clientelares resultan del amplio dominio que tuvieron, en las últimas décadas del siglo XX, los modelos de análisis de la elección racional (vg. Stokes et al., 2013). Estos análisis apelaron a los intereses egoístas como premisa ontológica de la naturaleza humana y, a partir de esa premisa, construyeron modelos para explicar cómo y por qué actúan los individuos involucrados en relaciones clientelares.

Asimismo, hacia fines de 1990, progresivamente comenzaron a tomar fuerza una serie de estudios etnográficos que problematizaron los supuestos de ambas literaturas. Estos trabajos hicieron valiosas críticas y aportes a ambas literaturas (eg. Quirós, 2011; Vommaro y Quirós, 2011). Sin embargo, casi no propusieron nuevos conceptos que den cuenta de modos concretos de participación política distintos de los señalados por las literaturas que critican. Esto se debería a que no lograron desembarazarse de los marcos conceptuales de las propias teorias sobre movimientos sociales y clientelismo. Tomadas como puntos de partida, dichas teorías recortan los límites de lo observable empíricamente a las formas de participación política y a los actores que usualmente contemplan. Como se verá, una combinación entre etnografía y teoría fundamentada no solo permite ampliar las concepciones que se tienen sobre los fenómenos del clientelismo y de los movimientos sociales, sino también el entendimiento que se tiene en general sobre la participación política de quienes residen en asentamientos informales mediante la elaboración inductiva de definiciones y caracterizaciones a partir de categorias guias (Glaser y Strauss, 1967). A continuación, se describen las etapas en que se dividió el proceso de investigación llevado a cabo y luego se repasan los resultados a los que se arribó. 


\section{Etnografia fundamentada: etapas y resultados}

Basado en la combinación de etnografia y teoría fundamentada, el proceso de investigación se dividió en cinco etapas: descubrimiento e investigación I, II, III y IV (todas ellas representadas en la figura 2). A estas se sumó una etapa de precampo, en la que se realizaron 220 encuestas a vecinos del asentamiento originalmente etnografiado, citadas en un apartado anterior. En su conjunto, el trabajo de campo se desarrolló en el periodo 2011-2015. Mientras la etnografía fundamentada en un asentamiento se hizo entre 2011 y 2014, la etapa de investigación IV desarrollada en otros asentamientos llevó un año más (2015). Por su parte, la redacción y difusión de resultados tomó otros dos años (2016-2017).

La etapa de descubrimiento comenzó con la elaboración de una categoría guía a partir de dos percepciones contrapuestas sobre la participación política: la primera, asociada a la búsqueda de bienestar social, igualdad o bien común y la segunda, a la lucha por el poder o por la dominación de los unos sobre los otros. Además, se excluyeron explícitamente de la categoría algunos ámbitos de acción. El resultado fue una categoría que refiere a la participación política en tanto acciones colectivas o individuales orientadas a la competencia por puestos de poder en el Estado y/o a resolver problemas sociales que afectan a uno o más individuos, con exclusión de las acciones o relaciones que se resuelven a través del mercado o por medio de conexiones familiares exclusivamente.

Partiendo de esa categoría guía, en la etapa de investigación I se elaboraron conceptos de baja abstracción que dieron cuenta de cuatro formas concretas de participación política no consideradas previamente por las literaturas sobre movimientos sociales y sobre clientelismo. Dichas categorias emergentes describieron procesos sociales básicos que sirvieron para continuar elaborando teoría (Holton y Glaser, 2012).

En la etapa de investigación II se arribó a un segundo tipo de aporte conceptual. Este consistió en una elaboración conceptual 
inductiva, de mayor grado de abstracción, a partir del análisis de las características comunes a las formas de participación política relevadas y conceptualizadas, y de su comparación con bibliografia elaborada previamente. Recuérdese que una vez elaboradas categorias emergentes que describen procesos sociales básicos, el conocimiento construido puede compararse con literatura ya hecha mediante una revisión bibliográfica. De ese modo, el proceso de investigación puede enriquecerse del conocimiento generado previamente, sin que este limite sus posibilidades de generar nuevo conocimiento.

En la etapa de investigación III, un nuevo descubrimiento abrió una línea de indagación no contemplada hasta el momento, sobre las formas en que el Estado hace efectiva su presencia en asentamientos informales y sobre las formas en que se relaciona con quienes residen allí. Producto de una nueva revisión bibliográfica y de los datos obtenidos en el campo, emergió una categoria de mayor grado de abstracción. En este punto, se dio por finalizado el trabajo etnográfico en el asentamiento analizado bajo el criterio de saturación teórica.

Finalmente, la etapa de investigación IV fue desarrollada mediante la realización de visitas etnográficas a otros tres asentamientos de la periferia de Buenos Aires localizados en regiones distintas (oeste, este y sur) a la región norte donde se localiza el asentamiento originalmente etnografiado. También se llevó a cabo una etnografía virtual de los sujetos abordados en el último; se realizaron entrevistas semiestructuradas a alrededor de 20 vecinos de otros ocho asentamientos y a 20 funcionarios públicos de todos los niveles de gobierno y se revisaron otras 40 entrevistas a vecinos de asentamientos y funcionarios públicos desarrolladas en el marco de proyectos afines. El cuestionario guía de las entrevistas elaboradas durante la etapa IV se confeccionó con base en las categorias emergentes desarrolladas en las etapas de investigación anteriores.

Respecto a los resultados del proceso de investigación, las cuatro formas de participación política construidas etnográficamente en la etapa de investigación I son: 1) prestación de servicios públicos; 
2) protestas sociales llevadas a cabo por brokers o punteros; 3) participación de vecinos en Espacios Estatales de Cooperación (de aquí en adelante EEC's), y 4) prestación de servicios públicos por parte de organizaciones financiadas por el Estado.

Mientras las primeras dos son formas desarrolladas por actores que fueron contemplados por las literaturas que estudian el clientelismo y los movimientos sociales, pero que, según los postulados de las mismas, no desarrollarian comúnmente ese tipo de acciones, las dos restantes son nuevas formas desarrolladas por actores que no fueron siquiera contemplados con antelación.

Las acciones que desarrolló un puntero llamado Báez con objeto de conseguir la provisión de agua potable para su barrio ilustran la categoría 1 . Pese a que los punteros solo se verían impulsados a intercambiar bienes privados por apoyo político como resultado de intereses egoístas, Báez fue el principal responsable de extender las redes de agua potable en su barrio para beneficio de todos sus vecinos, sean o no sus clientes. Para ello, apeló a sus contactos "en la política" y a su ascendencia sobre sus vecinos. Según sus palabras, esto le significó "muchos dolores de cabeza, pero valió la pena". En la siguiente cita, Báez explica cuánto deseaba la prestación del agua potable y cómo, cuando finalmente la consiguió, su ansiedad le impidió hacer el cálculo político que hizo su intendente de "esperar a los periodistas":

Yo siempre viví acá en el barrio y no se puede vivir sin agua [...] yo podía ir a bañarme a lo de un compañero del partido fuera del barrio y aun así sufría la falta de agua. ¿Te imaginás el resto de la gente? [...] Además, estaban todos los chiquitos enfermos, sucios [...]. Me acuerdo que cuando inauguramos la cañería central, el intendente me decía "espera que ya llegan los periodistas a sacar fotos", y yo le dije "¿Para qué quiero fotos? ¡Yo quiero el agua!".

Por su parte, distintas historias muestran cómo algunos sujetos suelen pasar de formar parte de organizaciones de movimientos sociales a redes clientelares, o viceversa; e intercalar participación en intercambios clientelares con la organización de protestas sociales. 
Este es el caso de Dalla, que ilustra la categoría emergente 2. Su historia se desarrolla del siguiente modo: primero formó parte de una organización de desocupados. Como integrante de esta, lideró una protesta para evitar que se les quitara la prestación del almuerzo a los niños que asistían a una escuela. Tras el éxito de su reclamo, se hizo conocida en el barrio y un candidato a intendente la incorporó a su partido para que obtuviera votos para su campaña. Por entonces, Dalla participó de intercambios clientelares coronando a dicho candidato como el intendente electo. Finalmente, ante la falta de respuestas estatales respecto de una serie de inundaciones que sufrió su barrio, Dalla volvió a organizar protestas, aunque esta vez frente a "su" propio intendente. Para explicar por qué llevó adelante una protesta frente al intendente para el que hizo campaña, Dalla apeló a una identidad distinta a la de puntera: "antes que todo, soy vecina".

Respecto a las categorías restantes, el activismo de un vecino sin pertenencia política llamado Garro, en cuanto EEC se desarrolló en su barrio, ilustra la categoría 3. Garro aprovechó cada instancia brindada por EEC's para promover un proyecto largamente mentado por él y por otros vecinos: construir un club social y deportivo que, además de tareas recreativas, brinde capacitación y contención a los niños del barrio.

Los EEC's son distintas instancias de participación que se llevan a cabo en asentamientos de la periferia de Buenos Aires desde 2008 (Techo, 2016), mediante reuniones periódicas entre vecinos y funcionarios en sedes de organizaciones barriales. Aunque el fin que declaran los EEC's es "apoyar" a los vecinos en la elaboración de proyectos y la búsqueda de soluciones a problemas, en la práctica se dedican a promover tareas de control social y a hacer pasar actividades partidarias como si fueran iniciativas de la comunidad (Besana, 2016). No obstante, la insistente participación de Garro torció la agenda no declarada de dichos espacios y logró imponer el proyecto del club. Además del esfuerzo que puso de su parte, Garro implementó una estrategia basada en la búsqueda de consensos entre vecinos y empleados públicos "de ventanilla", 
tales como docentes de escuelas y personal médico de salas de atención primaria. La cita que sigue muestra una de las tantas veces en que Garro intentó persuadir a quienes asisten a reuniones de EEC's de que su proyecto es de interés, no solo para los vecinos, sino también para todo aquel que desarrolla una labor social en el barrio:

Me parece que hacen falta organizaciones sociales que no estén tan ligadas a lo político para sacar a los pibes de los problemas de la calle $[\ldots]$ cuando los enganchás con algo que les gusta, como el fútbol o baile... les das oficios y capacitaciones que les sean útiles, les vas dando herramientas de trabajo para el futuro o para que no abandonan la escuela [...]. En el estatuto del club figuran unas cuestiones que incluyen todos los trabajos de los que están acá, y eso es lo que yo en el comienzo planteé para que todos los que se quieran sumar lo puedan hacer. Si eso llega a estar, obviamente van a estar todos con posibilidad de trabajar ahí, porque yo sé que hay un montón de actividades que se quieren hacer y que no se pueden hacer porque no hay un lugar físico [...] teniendo el club se pueden hacer un montón de actividades e incluir todas las tareas sociales que ustedes hacen...".

Como en el caso de la categoría que ilustra Garro, la categoría 4 muestra la participación de vecinos "de a pie" -sin pertenencia a un partido político o a una organización de movimientos sociales- que cumplen un rol fundamental a la hora de garantizar un mayor bienestar a sus vecinos. En este caso, dichos vecinos son "cooperativistas" beneficiarios de programas de transferencia condicionada y, como tales, se encargan de labores vinculadas a la prestación de bienes y servicios públicos. La historia de un cooperativista que debe reparar un tramo de la red de agua potable muestra cómo algunos de ellos deben "arreglárselas" para desarrollar sus labores, apelando a conocimientos y recursos propios e implementando distintas estrategias frente a los escasos recursos que les brinda el Estado para ello. En ese sentido, Espina "se las arregló" elaborando una serie de dibujos para suplir el desconocimiento de sus compañeros sobre los materiales con los que debían 
trabajar, para tener un mapa del barrio actualizado e incluso propuso un nuevo sistema de termo-fusión (ver figura 2), mejor adaptado a las características del suelo "con aguas emergentes" sobre el que se asienta el barrio, que el sistema propuesto por la propia agencia estatal de agua.

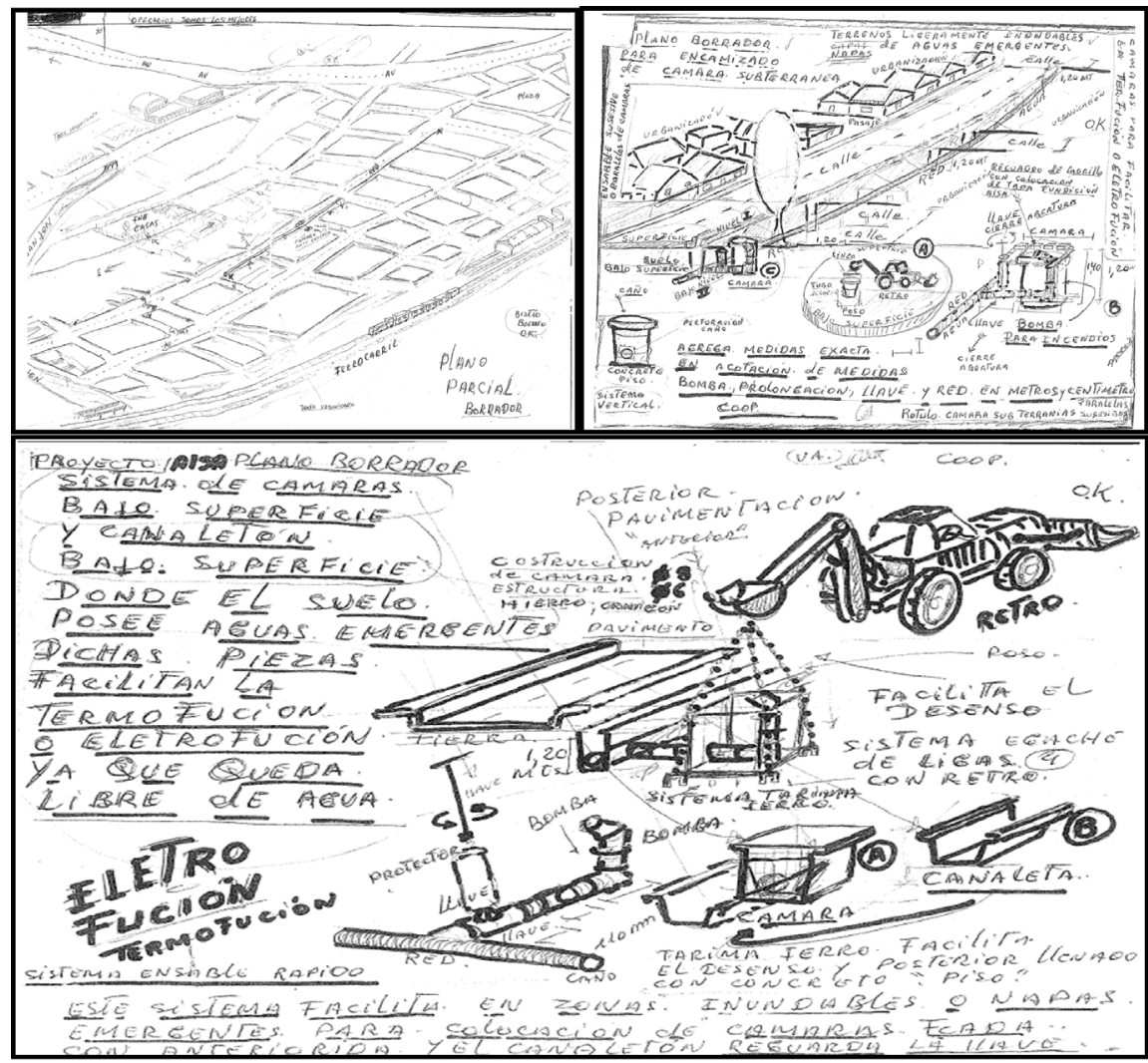

Figura 2. Mapa a mano de Santa María, plano borrador de sistema de cámaras y canaletón bajo superficie y plano borrador para encamisado de cámaras subterráneas elaborados por Espina

Fuente: copia del original.

Arribado a este punto, es de interés señalar cómo la estrategia etnográfica propuesta permitió mostrar: 1) que los punteros no solo son agentes partidarios, sino que también suelen ser vecinos de sus clientes y, como tales, manifiestan demandas y llevan a cabo acciones relacionadas con sus necesidades básicas, entre ellas la 
prestación de bienes y servicios públicos; 2) cómo las identidades políticas no son estáticas, ni excluyentes: un mismo sujeto puede ser puntero e integrante de una organización de movimientos sociales al mismo tiempo o en distintos momentos de su vida, y apelar a estrategias o acciones asociadas al clientelismo o a la protesta, y 3) cómo la tarea de los punteros no es sencilla, ni mecánica, sino que requiere de constancia y esfuerzo para obtener bienes y superar las dificultades que se presentan en el camino.

Respecto de las categorías 3 y 4, la estrategia de la "etnografia fundamentada" permitió: 1) posar los ojos en actores que no suelen ser percibidos por las literaturas sobre clientelismo y movimientos sociales; 2) describir cómo, en el desarrollo de distintas tareas, se convierten poco a poco en intermediarios entre el Estado y las necesidades de sus barrios, tales como los sujetos que sí contemplan dichas literaturas, y 3) cómo, a diferencia de los últimos, implementan otros tipos de recursos, conocimientos y estrategias para lograr sus cometidos.

Los resultados obtenidos en la etapa de investigación I fueron comparados entre sí y con los resultados obtenidos por otras literaturas, mientras se continuó desarrollando trabajo de campo. Fruto de una primera revisión bibliográfica llevada a cabo en la etapa de investigación II, surgieron varios elementos. Uno de ellos no es un resultado original del proceso de investigación, sino que tiene antecedentes en otras literaturas: la noción de intermediarios. Mientras la literatura sobre clientelismo utiliza dicho término como sinónimo de broker o puntero político, la de movimientos sociales lo hace -aunque en menor medida- para referirse a quienes coordinan y promueven la acción colectiva entre organizaciones sociales y/o agentes partidarios o estatales. Precisamente porque esta noción es utilizada por dichas literaturas, al tiempo que designa un elemento común a las cuatro formas de participación politica relevadas, se apeló a ella en la etapa de investigación II.

A partir de una definición más amplia de su significado, en tanto sujetos que se encuentran entre la esfera del Estado y el barrio, el concepto de intermediarios da cuenta de un aspecto común a la mayoría de las formas de participación política de quienes residen en 
asentamientos informales de la periferia de Buenos Aires; incluyendo tanto las formas contempladas por otras literaturas (clientelismo y movimientos sociales), como las que emergen de la presente propuesta. De ese modo, la etiqueta intermediarios contribuye a la elaboración de una visión amplia sobre la participación politica de estos sectores.

Tanto Báez y Dalla, en su carácter de punteros, como Garro, en tanto participante de EEC's, y Espina, como beneficiario de programas de transferencia condicionada, hacen presente al Estado en sus barrios a la par que comunican a este las necesidades de sus vecinos. En todos los casos, el trabajo que llevan adelante es doble. Así, por ejemplo, Báez convenció a sus jefes políticos para que lo ayuden a prestar el agua potable, negoció con representantes de distintas agencias y de otros partidos políticos para que no se lo impidan, y consiguió los recursos para llevar a cabo su cometido; pero también debió llevar a cabo "la otra parte del trabajo": convencer a sus vecinos para que caben las zanjas de sus casas, así como un gran pozo donde se instalaría la cañería central del sistema de agua. Algo similar sucedió con los restantes actores. De distintos modos, todos ellos manifiestan dos identidades: una referida a sus diversos vínculos con el Estado y la otra a su vínculo con el asentamiento. En otras palabras, todos ellos son medios de expresión del Estado en el barrio, al tiempo que satisfacen necesidades y canalizan demandas del barrio hacia el Estado.

Asimismo, un aspecto relacionado al concepto de intermediarios relativamente ausente en las teorías sobre la participación política de los segmentos más pobres, es el esfuerzo que deben realizar para satisfacer necesidades básicas (propias y/o de sus vecinos) y acceder a bienes y servicios de todo tipo. Un análisis más detallado del esfuerzo de los intermediarios decantó en la etapa de investigación III, en un descubrimiento sobre las formas que asume el Estado en asentamientos informales.

Allí se descubrió que el esfuerzo de los intermediarios es, en gran medida, resultado de un Estado que se ofrece a quienes residen en asentamientos informales, en el mejor de los casos, como 
"socio" o "sponsor" de sus esfuerzos. Esto no solo se observa en las formas en que se expresa el Estado, sino también en la concepción que los funcionarios públicos tienen respecto del deber del Estado en asentamientos. En la siguiente cita, un funcionario municipal justifica que la recolección de residuos en asentamientos sea llevada adelante por vecinos, sin otro equipamiento más que carros entregados por la municipalidad y no por la empresa que presta el servicio en el resto del distrito:

en asentamientos no recaudamos un solo centavo, esa gente no paga [...] entonces, nosotros prestamos un servicio solidario-distinto del que prestamos al contribuyente que paga una contraprestación- $[. .$.$] . Lo hacemos para que no genere olores y el contribuyente$ no se queje [...]. Los vecinos y las cooperativas de asentamientos tienen que hacerse responsables [...] si el servicio es de mala calidad es porque no se saben organizar para desarrollarlo de mejor manera [...]. Si tuvieran una organización del barrio e hicieran un esfuerzo, su barrio podría mejorar, como ha pasado con otros barrios $[. .$.$] . Los vecinos tienen que cooperar, juntarse entre sí y ver$ cómo pueden contribuir, los barrios siempre han crecido así, con base en la complementación entre la gente y el Estado.

Esta cita ilustra una concepción sobre el Estado distinta a la que tienen los mismos funcionarios respecto a los deberes del Estado para con "los contribuyentes". Así, mientras el Estado garantiza a sectores más acomodados acceso a bienes y servicios públicos -ya sea por medios propios o a través de empresas privadas-, en los asentamientos solo se ofrece como "socio" de los vecinos para que estos se procuren el acceso a dichos servicios mediante su propio esfuerzo. Si la provisión falla, es porque no hicieron el esfuerzo suficiente. En consecuencia, para dar cuenta de la lógica en que el Estado se hace presente en asentamientos, ya sea por medio de punteros, programas de transferencia condicionada, EEC's u otros canales que requieren de la participación de vecinos, se apeló el concepto Estado-socio (Grinberg et al., 2012; Osborne y Rose, 1999). ${ }^{4}$

4 Aunque dicha etiqueta ha sido utilizada en otros trabajos, su contenido difiere del que se le ha dado mediante el método propuesto. 


\begin{tabular}{|c|c|c|}
\hline $\begin{array}{c}\text { Etapas de } \\
\text { investigación }\end{array}$ & Categorias/Conceptos & $\begin{array}{c}\text { Nivel de } \\
\text { abstracción }\end{array}$ \\
\hline $\begin{array}{c}\text { Etapa de } \\
\text { descubrimiento }\end{array}$ & $\begin{array}{c}\text { Categoria guía: Participación } \\
\text { politica }\end{array}$ & \\
\hline
\end{tabular}

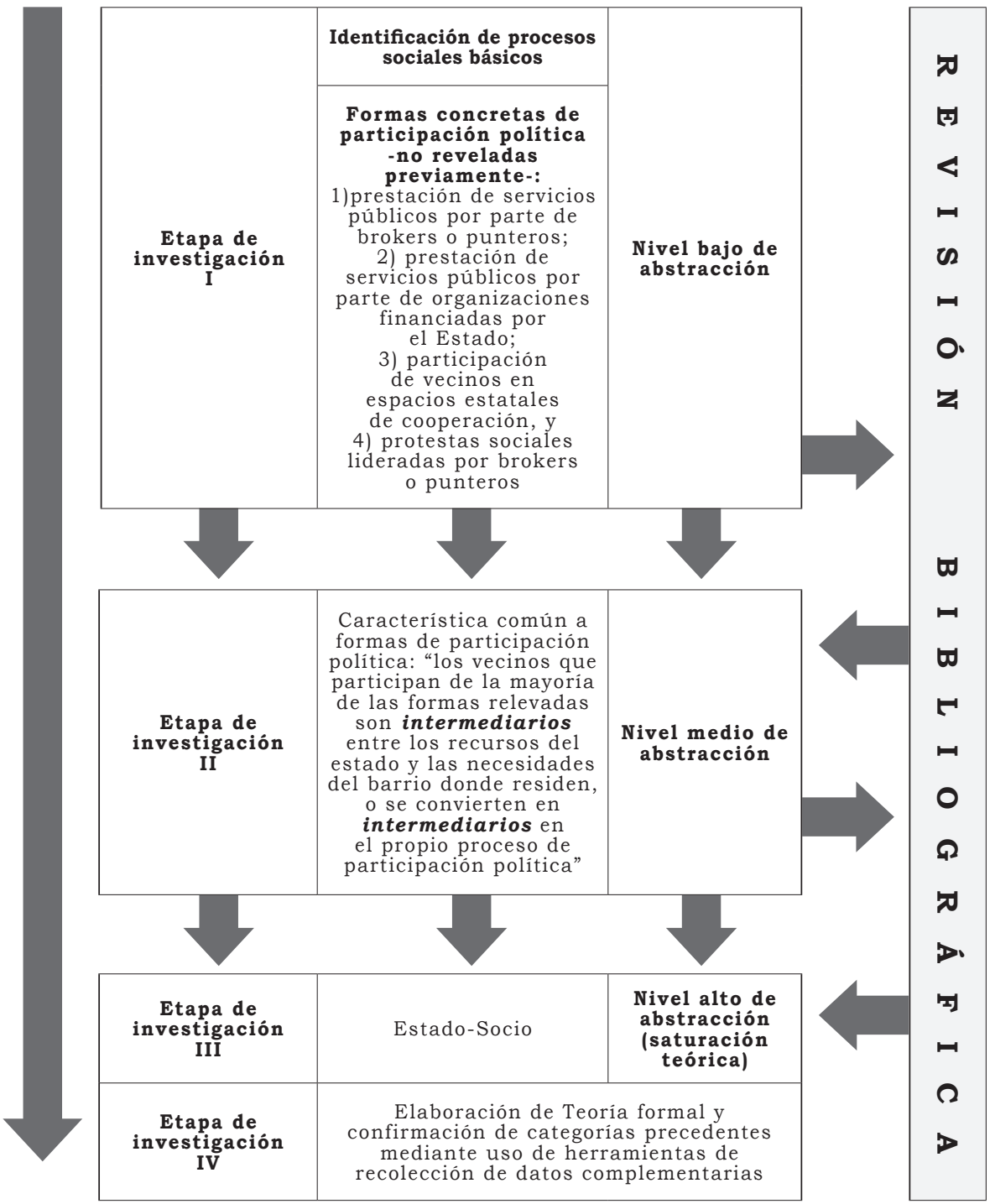

Figura 3. Etapas de investigación, categorías y nivel de abstracción Fuente: elaboración propia. 
Teóricamente, el Estado-socio es el reverso de la participación política de intermediarios en asentamientos informales. Dicho de otra manera, el Estado se hace presente en asentamientos como "socio" de los vecinos, que se convierten en intermediarios entre los recursos del Estado y las necesidades del barrio donde residen. De ese modo, la noción intermediarios revela una característica de la participación política en asentamientos vinculada a las formas que asume el Estado allí, es decir al Estado-socio, y el Estado-socio recorre el camino de vuelta: explica en gran medida por qué los vecinos participan de las formas en que participan.

Sometido a una nueva revisión bibliográfica -esta vez sobre el Estado en asentamientos- se observó que el Estado-socio describiría la cotidianeidad de los contextos analizados per se. En cambio, otras explicaciones más populares resaltan el carácter represivo que asume el Estado en situaciones excepcionales; o -desde una perspectiva más institucionalista- hacen hincapié en la falta o inestabilidad institucional del Estado en asentamientos respecto de territorios donde residen sectores más acomodados (Levitsky, 2001; Davis, 2007; Gupta, 2012). En ese sentido, la explicación del Estado-socio es complementaria a las otras dos y contribuye a tener una noción más general sobre la relación Estado/sociedad en asentamientos informales.

Puesto que la información recabada durante la etapa de investigación III, no reportó más conocimiento nuevo se consideró que el proceso de investigación alcanzó su saturación teórica. De ese modo, la categoria Estado-socio, fue la de mayor grado de abstracción entre las elaboradas. Construida por inducción y por agregación, comprende a las restantes categorias emergentes. En sí, aporta una visión amplia sobre las relaciones Estado/sociedad en asentamientos y sobre las formas en que participan quienes residen allí, incluyendo aportes teóricos de las literaturas sobre clientelismo y movimientos sociales.

La etapa de investigación IV se desarrolló con objeto de poner a prueba las categorias elaboradas mediante un análisis de caso 
en otros casos y reforzar la robustez de la evidencia elaborada etnográficamente por medio del uso de otras herramientas. Por su parte, el desarrollo de una etnografía virtual no fue planeado, aunque resultó de la cercanía entre investigador y sujeto abordado que propone la etnografia offline. Hacia el final de la investigación, muchos sujetos ofrecieron su amistad en la red Facebook a quien subscribe. La constatación de que ellos y muchos otros tenían perfiles en dicha red y que allí compartían acciones y campañas de interés para el proyecto de investigación motivó la inclusión de la etnografia virtual en el mismo.

En general, los resultados de las visitas etnográficas y de las restantes herramientas confirmaron el contenido de las categorias emergentes, sin agregar nuevo conocimiento. De esto podría deducirse que: las restantes herramientas reforzaron la robustez de las categorías elaboradas etnográficamente; o bien, que las restantes herramientas tienen grandes dificultades para generar nuevo conocimiento en contextos como el analizado. Ya sea por la positiva o por la negativa, ambas opciones otorgarian cierto aval a la perspectiva etnográfica aquí propuesta para la elaboración de una teoría formal amplia sobre la participación política de sujetos socialmente situados.

Por su parte, la etnografia virtual mostró resultados que ponen en entredicho algunas de las categorias elaboradas offline. A diferencia del análisis territorialmente situado, la etnografia virtual centra su atención en el seguimiento de relaciones y conexiones online-offline (Hine, 2004). A partir de un análisis de la red social Facebook, se pudo observar que las relaciones y publicaciones de algunos sujetos abordados en ocasiones excedian las fronteras del asentamiento donde residen. Su alcance por fuera de los límites del barrio supone una innovación que podría incrementarse con el tiempo. La realización de acciones de deslocalización o desterritorialización por parte de actores cuyas relaciones sociales y politicas se enmarcan fuertemente en el territorio (Merklen, 2005) podría suponer una serie de cambios respecto de sus formas de participación y de su relación con el Estado en el plano 
offline. Esto amerita el desarrollo de nuevos estudios que analicen en profundidad la participación online-offline de estos sujetos. Hasta tanto eso suceda, el concepto Estado-socio y la noción de intermediarios aquí presentada, brindan una visión amplia, que incluye los aportes teóricos de otras literaturas, sobre las relaciones Estado/sociedad en asentamientos y las formas que se participa políticamente allí.

\section{Referencias}

Almond, G. y Verba, S. (1963). Civic Culture. Londres: Sage.

Balbi, F. A. y Boivin, M. (2008). La perspectiva etnográfica en los estudios sobre política, Estado y gobierno. Cuadernos de Antropología Social, (27), 7-17.

Bauböck, R., Della Porta, D., Lago, I. y Ungureanu, C. (2012). ¿De las "guerras" metodológicas al pluralismo metodológico? Revista Española de Ciencia Política, (29), 11-38.

Box-Steffensmeier, J., Brady, E. y Collier, D. (Eds.) (2008). The Oxford Handbook of Political Methodology. Oxford: Oxford University Press.

Collier, D. y Elman, C. (2008). Qualitative and multi-method research: Organizations, publication, and reflections on integration. En J. M. BoxSteffensmeier, H. E. Brady y D. Collier (Eds.), The Oxford Handbook of Political Methodology (780-795). Oxford: Oxford University Press.

Collier, R. B. y Handlin, S. (2009). Reorganizing Popular Politics: Participation and the New Interest Regime in Latin America. Pensilvania: The Pennsylvania State University Press.

Cravino, M. C., Del Río, J. y Duarte, J. (2007). Magnitud y crecimiento de las villas y asentamientos en el Área Metropolitana de Buenos Aires en los últimos 25 años. Buenos Aires: Universidad Nacional de General Sarmiento. 
Cuñat Giménez, R. J. (2007). Aplicación de la teoría fundamentada (grounded theory) al estudio del proceso de creación de empresas. En Decisiones basadas en el conocimiento y en el papel social de la empresa. XX Congreso anual de AEDEM (Comunicaciones). España: Asociación Española de Dirección y Economía de la Empresa (AEDEM).

Curutchet, G., Grinberg, S. y Gutiérrez, R. A. (2012). Degradación ambiental y periferia urbana: un estudio transdiciplinario sobre la contaminación en la Región Metropolitana de Buenos Aires. Revista Ambiente \& Sociedade, 15(2), 173-194.

Davis, M. (2007). Planet of Slums. London y Brooklyn: Verso.

Defensor del Pueblo de la Nación, FARN, Fundación Protigre y Cuenca del Plata. (2007). Informe especial Cuenca del Río Reconquista primera parte. Buenos Aires: Defensoría del Pueblo de la Nación.

Della Porta, D. y Keating, M. (2008). Approaches and methodologies in the Social Sciences: a pluralist perspective. Cambridge: Cambridge University Press.

Flick, U. y Maeder, C. (2005). Qualitative Methods in Europe: The Variety of Social Research. Special issue of Forum Qualitative Sozialforschung / Forum: Qualitative Social Research, 6(3), Art. 34.

Gerber, A., Green, D. y Kaplan, E. (2014). The Illusion of Learning from Observational Research. En I. Shapiro, R. Smith y T. Masoud (Eds.), Problems and Methods in the Study of Politics (pp. 251-273). Cambridge: Cambridge University Press.

Glaser, B. y Strauss, A. (1967). El desarrollo de la teoría fundada. Chicago: Aldine.

Grinberg, S., Gutiérrez, R. y Mantiñan, L. (2012). La comunidad fragmentada: gubernamentalidad y empoderamiento en territorios urbanos hiperdegradados. Revista Espacios Nueva Serie, (1), 154-172. 
Guber, R. (2001). La etnografia. Método, campo y reflexividad. Buenos Aires: Norma.

Gupta, A. (2012). Red Tape: Bureaucracy, Structural Violence, and Poverty in India. North Carolina: Duke University Press.

Herzfeld, M. (2004). Cultural Intimacy: Social Poetics in the Nation-State. Nueva York y Londres: Routledge.

Hine, C. (2004). Etnografia virtual (Colección Nuevas Tecnologias y Sociedad). Barcelona: Editorial UOC.

Holton, J. y Glaser, B. (2012). The Grounded Theory Review Methodology Reader: Selected Papers 2004-2011. Mill Valley: Sociology Press.

King, G., Keohane, R. O. y Verba, S. (2000). El diseño de la investigación social: la inferencia cientifica en los estudios cualitativos. Madrid: Alianza.

Levitsky, S. (2001). An Informal Organisation and the Persistence of Local Party Structures in Argentine Peronism. Journal of Latin American Studies, 33(01), 29-65.

Merklen, D. (2005). Pobres ciudadanos: las clases populares en la era democrática, Argentina, 1983-2003. Buenos Aires: Editorial Gorla.

Migdal, J. S. (2009). State in Society: Studying How States and Societies Transform and Constitute One Another. Cambridge - New York: Cambridge University Press.

Murillo, V. y Ramos, G. (2017). La contextualización en la ciencia política: una perspectiva latinoamericana. Foro Internacional, 55(220), 576-594.

Osborne, T. y Rose, N. (1999). Governing Cities: Notes on the Spatialisation of Virtue. Environmental and Planning D: Society and Space, (17), 737-760.

Panebianco, A. (1990). Modelos de partido: organización y poder en los partidos políticos. Madrid: Alianza. 
Pasquino, G. (2011). Nuevo curso de ciencia politica. México D. F.: Fondo de Cultura Económica.

Piven, F. F. y Cloward, R. (2012). Poor People's Movements: Why They Succeed, How They Fail. New York: Knopf Doubleday Publishing Group.

Pizzorno, A. (1975). Introducción al estudio de la participación política. En A. Pizzorno, M. Kaplan y M. Castells (Eds.), Participación y cambio social en la problemática contemporánea (13-82). Buenos Aires: Ediciones Siap-Planteos.

Quirós, J. (2011). El clientelismo como incógnita. Antropólogos, sociólogos y politólogos. Desarrollo Económico, 50(200), 631-641.

Schatz, E. (Ed.). (2009). Political ethnography: What immersion contributes to the study of power. Chicago: University of Chicago Press.

Shapiro, I., Smith, R. y Masoud, T. (Eds.) (2004). Problems and Methods in the study of Politics. Cambridge: Cambridge University Press.

Stokes, S., Dunning, T., Nazareno, M. y Brusco, V. (2013). Brokers, Voters and Clientelism. The Puzzle of Distributive Politics. New York: Cambridge University Press.

Svampa, M. y Pereyra, S. (2005). La política de los movimientos piqueteros. En F. Schuster (Ed.), Tomar la palabra: estudios sobre protestas sociales y acción colectiva en la Argentina contemporánea (343-364). Buenos Aires: Prometeo.

Techo. (2016). Relevamiento de asentamientos informales 2016. Techo, Argentina: Techo.

Tilly, C. (2008). Contentious Performances. Cambridge: Cambridge University Press.

UN-Habitat. (2012). Estado de las ciudades de América Latina y el Caribe 2012: rumbo a una nueva transición urbana. San Pablo: UN - Habitat. 
Wacquant, L. (2007). Los condenados de la ciudad. Gueto, periferias y Estado. Buenos Aires: Siglo XXI.

Weeden, L. (2012). Reflections on ethnographic work in political science. Annual Review of Political Science, (13), 255-272.

Vommaro, G. y Quirós, J. (2011). “Usted vino por su propia decisión”: Repensar el clientelismo en clave etnográfica. Desacatos, (36), 65-84.

Zarazaga, R. (2013). Vote-Buying and Asymmetric Information. A Model with Applications to Argentina. Working paper 398. Notre Dame, Indiana, EE.UU.: The Kellogg Institute.

\section{Cómo citar este artículo}

Besana, P. B. (2018). Notas sobre el uso de la etnografia y la teoria fundamentada en ciencia política. Un análisis amplio de la participación política y el Estado en asentamientos informales de la periferia de Buenos Aires, Argentina. Universitas Humanistica, 86, 107-137. https://doi.org/10.11144/Javeriana.uh86.nuet 